\title{
Modelación matemática de bibliotecas en desarrollo
}

\author{
EMILIO SETIÉN QUESADA \\ InvestigadortitulardelaBiblio tecaNacio nalJoséMartí, \\ PlazadelaRevo lu ción, AP 688, LaHabana, Cuba \\ Tel. 8191 11, Télex 51 19 63; Fax 816224 \\ E-mail:setien@ binanet.cu
}

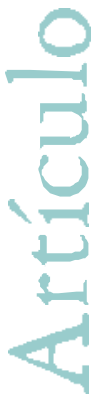

\begin{abstract}
RESUMEN
Se hace referencia a dos artículos sobre el tema, publicados por el autor en Investigacón biblictecdógica. El aparecido en el v. 5 no. 11 de 1991, donde se explican las características de las bibliotecas en desarrollo que aconsejan el empleo de números índice en la modelación de su comportamiento. El incluido en el v. 9 no. 19 de 1995, que contiene el modelo de comportamiento de las bibliotecas públicas cubanas, los presupuestos bibliotecológicos que lo sustentan y los antecedentes directos que se le reconocen. Se ofrece ahora, como complemento de esa información, los presupuestos matemáticos tomados en consideración y el análisis de otros ejemplos de números índice aplicados a la actividad bibliotecaria, localizados durante la investigación, pero que no se tomaron como antecedentes del modelo cubano.
\end{abstract}

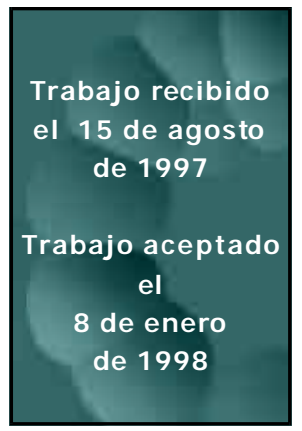

\section{ABSTRACT}

Reference is made to two papers on the subject published by the authoria Investigaciones Bibliotecológicas (Library Science Research). The first which appeared in vol. 5. No. 11, 1991, explains the features of developing libraries and advises on the use of index numbers in the modeling of their behaviour. The second, included in vol. 9 No. 19, 1995, contains a behaviour model for public libraries in Cuba, the librarianship principles and previws knowledge which sustain it. To complement this information, the mathematical assumptions used are now presented as well as the analysis of other examples of index numbers applied to library science. These examples were not taken into consideration in the development of the Cuban model.

\footnotetext{
Como fue expuesto por quien suscribe en artículo publicado en InvestigaciónBiblidedóǵca, v. 5 no. 11 julio-diciembre de 1991, el comportamiento de las bibliotecas en desarrollo - ejemplificado con el caso de las bibliotecas públicas cubanas- , se caracteriza por ser un proceso no estabilizado en el que pesa más la singularidad en el tiempo y el espacio que la generalidad, lo que limita la posibilidad de aplicar métodos propios de la modelación de tendencias y de la elabora-
} 
ción de pronósticos. En el propio artículo se explica cómo, ante una situación así, lo aconsejable es encauzar el análisis hacia modelos que permitan obtener generalizaciones sobre lo ya ocurrido en esas instituciones y que sirvan para comparar unas bibliotecas con otras, o la actuación de cada una de ellas en distintos periodos. Finalmente, el artículo antes mencionado sustenta la conveniencia de aplicar números índice a la modelación de ese comportamiento.

En otro trabajo, publicado también en Investigacoón Biblidteedógica (v. 9, no. 11 julio-diciembre de 1995), se explica la representación de los elementos y relaciones esenciales de la actividad bibliotecaria en términos de eficiencia y se exponen los presupuestos bibliotecológicos que sustentan los diversos índices que simbolizan esas relaciones. En ese artículo se presenta también el modelo adoptado para las bibliotecas públicas cubanas y sus antecedentes directos.

Ahora se ofrecen los presupuestos matemáticos que orientaron el trabajo realizado y el análisis de dos ejemplos más de números índice aplicados a la actividad bibliotecaria, que fueron localizados en la literatura consultada. Si bien estos ejemplos no fueron tomados como antecedentes del modelo cubano, sí muestran la forma de utilizar números índice en la representación del trabajo bibliotecario.

La matemática se define como la ciencia cuyo objeto de estudio comprende cualesquiera formas de la realidad, que posean objetivamente un grado tal de independencia del contenido, que puedan ser abstraídos de él; comprende, asimismo, el estudio de los propios objetos matemáticos y de las relaciones entre ellos, desde el punto de vista abstracto. ${ }^{1}$

En ese contexto, los modelos matemáticos son prototipos de forma idealizada, objetos abstractos que, por analogía, representan un proceso o fenómeno, aunque reflejen solamente las características más importantes, entre las que deben estar presentes, directa o indirectamente, las que sean esenciales para el fenómeno estudiado. El modelo matemático es una estructura abstracta en la cual los objetos de la realidad, y las relaciones entre ellos, son sustituidos por objetos y relaciones matemáticas. Es frecuente que los objetos y relaciones matemáticas que conforman esos modelos expresen características susceptibles de medición en los objetos y relaciones de la realidad que sustituyen. Tal ha sido la dirección principal de la matematización del conocimiento científico, puesto que en la aplicación de la matemática a las ciencias naturales, técnicas y sociales, el papel determinante lo desempeñan los modelos que se basan en la medición, aunque no son los únicos. Teniendo en cuenta el carácter de las leyes a que se someten los fenómenos estudiados, los modelos matemáticos se dividen en tres grupos: aquellos que modelan objetos que obedecen a leyes dinámicas, los que representan objetos que responden a leyes estadísticas y aquellos que modelan objetos en cuyo

1 Academia de Ciencias de la URSS. Instituto de Filosofía. La dialéticaylos mútodos äentíficos genera les deinvestigaaión Ob. Cit. En bibliografía, t. 1. p. 187. 
comportamiento no se identifica la acción de leyes, por lo que representan situaciones indeterminadas. ${ }^{2}$

El modelo matemático es, pues, una estructura abstracta que representa la forma de los objetos de la realidad y las relaciones concretas que existen entre ellos, mediante la selección de aquellos elementos que responden a las características esenciales del objeto o fenómeno estudiado, simbolizados matemáticamente -de forma directa o indirecta- y expresados, mayormente, en términos asequibles a la medición, que permiten representar comportamientos concretos, puntuales, o en forma de tendencias.

$\mathrm{Y}$, precisamente, los números índice sirven para representar comportamientos puntuales y compararlos entre sí, aunque no se identifiquen en ellos, necesariamente, tendencias que acusen la acción de leyes dinámicas o estadísticas.

En la literatura consultada durante la investigación realizada para diseñar el modelo de comportamiento de las bibliotecas públicas cubanas aparecen varios métodos matemáticos utilizados para reflejar situaciones concretas de elementos y relaciones propios de la actividad bibliotecaria. Entre ellos existen algunos modelos que ofrecen, al mismo tiempo, índices integrales de comportamiento de las bibliotecas, que pueden ser empleados para compararlas entre sí o para comparar la actuación de cada una de ellas en distintos periodos. En el artículo publicado en Inestigadónbiblictecóógica del año 95, se analizaron los empleados por N.A. Rubakin y E. N. Medinski y por P. V. Rzasa y N. R. Baker, por constituir antecedentes directos del modelo arriba mencionado. ${ }^{3}$

A hora se explican otros dos ejemplos que, aunque no tienen esa condición, sí evidencian la forma de utilizar números índice en el caso que se analiza.

\section{EL COEFICIENTE DE INTENSIDAD DE LA ACTIVIDAD BIBLIOTECARIA EMPLEADO POR A. BALIKA ${ }^{4}$}

Este coeficiente no difiere esencialmente del empleado por Rubakin y $\mathrm{Me}$ dinski y es posterior al de estos autores. Sólo se distingue de aquél por la manera de presentar la fórmula del coeficiente:

$$
\mathrm{CI}=\frac{\mathrm{m}^{2}}{\mathbb{N}}
$$

donde

2 LuisaRedon do. Estadísticas para lasinuetigariones soidógicas O b. cit. en bibliografía, p. 12, 23.

3 I. M. Frumin. Organizacón de trabajodela biblictea saiếica Ob. cit. en bibliografía, p. 162. Rzasa, Philip V. y Nor man R. Ba ker. MeasuresofEffectivenessforaUnivensityLibrary. O b. cit. en bibliografía p. 248-253.

4 I. M. Frumin, Ob. cit. p. 163. 
CI es el coeficiente de intensidad, mes la cantidad de préstamos; $f$ la cantidad de documentos en la colección; $\mathrm{N}$, la cantidad de lectores potenciales.

Para Rubakin y Medinski:

$$
\mathrm{CI}=\mathrm{C} \cdot \mathrm{R}
$$

pero como el índice de circulación C es el promedio de préstamos (m) por lector potencial $(\mathrm{N})$

$$
\mathrm{C}=\frac{\mathrm{m}}{\mathrm{N}}
$$

y el de rotación $\mathrm{R}$ es el promedio de préstamos (m) por documento en colección (f)

$$
\mathrm{R}=\frac{\mathrm{m}}{\mathrm{f}}
$$

entonces:

$$
\mathrm{CI}=\left(\frac{\mathrm{m}}{\mathrm{N}}\right)\left(\frac{\mathrm{m}}{\mathrm{f}}\right)=\frac{\mathrm{m}^{2}}{\mathrm{fN}}
$$

o sea, la fórmula empleada por Balika.

En tanto no existan diferencias esenciales entre la fórmula de Balika y la empleada por Rubakin y Medinski, el análisis crítico presentado en artículos anteriores sobre el coeficiente de intensidad empleado por esos autores, será válido también para el de Balika. Debe señalarse, no obstante, que el tratamiento de las variables en la fórmula de Balika, aunque matemáticamente más acabado, es menos expresivo (informativo) que el dado por Rubakin y Medinski. En la fórmula de estos últimos se puede identificar directamente el comportamiento de los índices de circulación y rotación, lo que contribuye a obtener conclusiones sobre la frecuencia de lectura de los usuarios y sobre el nivel de uso de las colecciones. En la fórmula de Balika esto no es posible. 


\section{EL ÍN DICE DE AMPLIT U O ÓPTIMA DE LAS COLECCIONES DE JU. N. ST OLJAROV ${ }^{5}$}

Este índice constituye básicamente una aplicación del índice de rotación (cfr. ejemplo anterior), aunque en el planteamiento de su fórmula, el autor utiliza el índice de circulación (cfr.) y el de alcance de las colecciones (que se explica de inmediato) para representar el índice de rotación.

La fórmula es la siguiente:

$$
\mathrm{E}=\frac{\mathrm{C}}{\mathrm{D}}
$$

donde

E es el índice de amplitud óptima (rotación), $\mathrm{C}=\frac{\mathrm{m}}{\mathrm{N}}$ es el índice de circulación ( $\mathrm{m}=$ préstamos, $\mathrm{N}=$ lectores potenciales) y $\mathrm{D}=\frac{\mathrm{f}}{\mathrm{N}}$ es el índice de alcance de la colección ( $\mathrm{f}=$ cantidad de documentos en colección, $\mathrm{N}$ = lectores potenciales).

Puede demostrarse que el valor de E expresa el índice de rotación

$$
\mathrm{R}=\frac{\mathrm{m}}{\mathrm{f}}
$$

puesto que;

$$
\begin{gathered}
E=\frac{C}{D}=\frac{m}{N}= \\
\frac{m}{\frac{f}{N}} \cdot f=\frac{m}{f}=R
\end{gathered}
$$

No obstante esta identidad, el autor propone la fórmula

$$
\mathrm{E}=\frac{\mathrm{C}}{\mathrm{D}}
$$

puesto que ésta permite observar y comparar la frecuencia de préstamos por lector con la cantidad de volúmenes en colección por lector y con el propio promedio de préstamos por documento en colección, factores entre los cuales Stol-

5 Ju. N. Stoljarov, Amplitud q́dima delos fondos delas bibidteeas púbicas Ob. cit. en bibliografía, p. 23-29. 
jarov asume que existen determinadas relaciones causa-efecto, que analiza detenidamente en su trabajo.

En el modelo de Stoljarov, como en los del coeficiente de intensidad de los autores analizados anteriormente, están ausentes: el rendimiento de los bibliotecarios, la relación entre los lectores que utilizan las colecciones y los que están inscritos en las bibliotecas, así como entre éstos y los lectores potenciales. No obstante, en este caso aparece una relación que no tienen en cuenta las fórmulas anteriores: el índice de documentos por lector potencial (D) que sirve, como ya quedó expresado, para definir el alcance de las colecciones de las bibliotecas.

A continuación se ofrece una tabla que muestra, en síntesis, los modelos comentados en este trabajo y en los artículos que lo preceden, a los que se hizo oportuna referencia.

\begin{tabular}{|c|c|c|c|c|c|}
\hline \multirow[t]{2}{*}{ ELEMEN TOS } & \multicolumn{5}{|c|}{ MODELOS } \\
\hline & $\begin{array}{l}\text { Rubakin y } \\
\text { Medinski }\end{array}$ & Balika & Stoljarov & $\begin{array}{c}\text { Rzasa y } \\
\text { Baker }\end{array}$ & $\begin{array}{l}\text { Modelo } \\
\text { cubano }\end{array}$ \\
\hline Préstamos (m) & $\mathrm{X}$ & $\mathrm{X}$ & $\mathrm{X}$ & $\mathrm{X}$ & $\mathrm{X}$ \\
\hline Colección (f) & $\mathrm{X}$ & $\mathrm{X}$ & $\mathrm{X}$ & 0 & $\mathrm{X}$ \\
\hline Lector potencial $(\mathrm{N})$ & $\mathrm{X}$ & $\mathrm{X}$ & X & $\mathrm{X}$ & $\mathrm{X}$ \\
\hline Lector inscrito (n) & 0 & 0 & 0 & $\mathrm{X}$ & $\mathrm{X}$ \\
\hline $\begin{array}{l}\text { Lector que utiliza } \\
\text { colecciones(I) }\end{array}$ & 0 & 0 & 0 & 0 & $\mathrm{X}$ \\
\hline Bibliotecarios $(\mathrm{B})$ & 0 & 0 & 0 & 0 & 1 \\
\hline \multicolumn{6}{|l|}{ RELACIONES } \\
\hline Circulación (C) & $\mathrm{X}$ & $\mathrm{X}$ & $\mathrm{X}$ & $\mathrm{X}$ & $\mathrm{X}$ \\
\hline Rotación (R) & $\mathrm{X}$ & $\mathrm{X}$ & $\mathrm{X}$ & 0 & $\mathrm{X}$ \\
\hline \multicolumn{6}{|l|}{ Alcance de: } \\
\hline Comunicación (a) & 0 & 0 & 0 & 0 & $\mathrm{X}$ \\
\hline Captación (ai) & 0 & 0 & 0 & $\mathrm{X}$ & $\mathrm{X}$ \\
\hline Colección (D) & 0 & 0 & X & 0 & 2 \\
\hline Productividad & 0 & 0 & 0 & 0 & $\mathrm{X}$ \\
\hline \multicolumn{6}{|c|}{$\begin{array}{l}\mathrm{x}=\text { presen ciadeelemen to o relación } \\
0=\text { ausencia de elemento o relación } \\
1 \text { = presente, in directamen te, en lospro mediosdecadaelemen to porbiblio tecario, como } \\
\text { expresióndeproductividad } \\
2=\text { presente, in directamen te, en tan to el número delec to respo ten cialessedeterminaapartir } \\
\text { de la colecciónexistente y del per cápitade volúmenes por lector potencialconsiderado en las } \\
\text { políticas de las bibliotecas públicas cubanas }\end{array}$} \\
\hline
\end{tabular}




\section{FóRMULAS}

Rubakin y Medinski

$$
\mathrm{CI}=\mathrm{C} \cdot \mathrm{R}
$$

Balika

$$
\mathrm{CI}=\frac{\mathrm{m}^{2}}{\mathrm{NF}}
$$

Stoljarov

$$
\mathrm{E}=\frac{\mathrm{C}}{\mathrm{D}}
$$

Rzasa y Baker

$$
\mathrm{C}=\frac{\mathrm{m}}{\mathrm{N}}\left(1+\frac{\mathrm{n}}{\mathrm{N}}\right)
$$

Modelo cubano

$$
\mathrm{IC}=\sqrt{\frac{\mathrm{m} / \mathrm{f} \cdot \mathrm{m} / \mathrm{h}(1+1 / \mathrm{n})(1+\mathrm{n} / \mathrm{N})}{\mathrm{m} / \mathrm{fi} \cdot \mathrm{m} / \mathrm{li}(1+\mathrm{li} / \mathrm{ni})(1+\mathrm{n} / \mathrm{Ni})}}
$$

Entonces el modelo cubano es más completo, porque en los antecedentes localizados:

a) no se emplean todos los elementos y relaciones identificados como componentes del concepto de eficiencia de las bibliotecas, ya sea de forma directa 0 indirecta

b) no expresan el nivel preciso de la comunicación social autor - lector que se logra en las bibliotecas

c) no consideran el papel del bibliotecario en el desarrollo de la actividad, por lo que escapa a la modelación un elemento, que junto con los lectores, constituyen los agentes activos principales que intervienen en el desarrollo de esa actividad

d) no ofrecen un índice que sintetice el comportamiento comparado de lugares y periodos.

Finalmente, ha de tenerse en cuenta que las posibilidades de aplicar estos índices depende del sistema de control de datos estadísticos empleado por las bibliotecas; de su adecuación al fenómeno que reflejan, de su utilización práctica, compatibilidad y confiabilidad. 


\section{BIBLIOGRAFÍA}

A cademiadeCien cias dela URSS. Instituto deFilo so fía.Ladialéticaylosmátodosaientíficos generalesdinvestigadón Mi jail E. O ne lia novsky. et. al. tr. de Félix de la Uz. La Habana: Editorial de Ciencias Sociales, 1981. 2 t. (Filosofía)

Frumin, I.M. Organización de trabajo dela biblictea saviética 1981. 170 h. Mecanografiado. Traducido del ruso. Publicado como: Organizatsiarabotisoveskoibibliotdki. Moskva: Kniga, 1969. 280 s.

Redondo, Luisa. Estadística para las investigadiones socidógicas / La Habana: Ministerio de Educación Superior 198-/ - 420pp.

Rzasa, Philip V. and Norman R. Baker. "Measures of Effective ness for a University Library." Jaumal of theAmmican Socity for Infomation Saiences(Washington) 23 (4): 248-253; July-A ugust, 1972.

Setién Quesada,Emilio. "Estado deD esarrollo delasbiblio tecas públicas cubanas.” Investigacón Bibliotedógica (México, D.F.) 5 (11): 24-32; julio-diciembre, 1991.

- -. "Modelo de comportamiento de las bibliotecas públicas cubanas y su índice representativo." Investigacón Biblidtedớġa (México, D .F.) 9(19): 12-16; julio-diciembre, 1995.

Stoljarov, Ju. N. "Amplitud óptima de los fon dos delas bi blio tecas públicas." Bdén dela UNESCO para las biblicteras (París) 27(1): 23-29; enero-febrero, 1973. La Habana, 28 de abril de 1997.

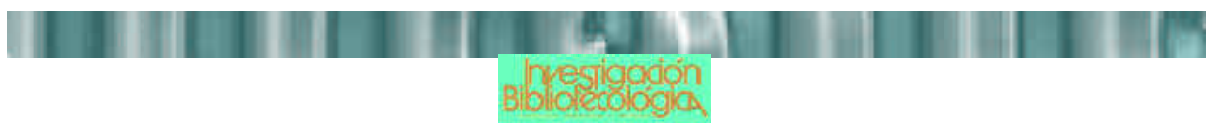

